\title{
Quantum enhanced joint measurement of two conjugate observables with an $\mathrm{SU}(1,1)$ interferometer
}

\author{
Yuhong Liu ${ }^{1}$, Jiamin $\mathrm{Li}^{1}$, Nan Huo ${ }^{1}$, Xiaoying $\mathrm{Li}^{1}$, Z. Y. Ou ${ }^{2}$ \\ ${ }^{1}$ College of Precision Instrument and Opto-Electronics Engineering, Key Laboratory of Opto-Electronics Information \\ Technology, Ministry of Education, Tianjin University, Tianjin, 300072, China \\ 2 Department of Physics, Indiana University-Purdue University Indianapolis, Indianapolis, IN 46202, USA \\ xiaoyingli@tju.edu.cn, zou@iupui.edu
}

\begin{abstract}
We jointly measure the phase and amplitude modulation of an optical field with the newly developed $\mathrm{SU}(1,1)$ interferometer. We simultaneously achieve a signal-to-noise ratio improvement of 1.1 and $1 \mathrm{~dB}$ over the standard quantum limit in amplitude and phase measurement.
\end{abstract}

Keywords-quantum metrology; joint measurement; nonlinear interferometer

Heisenberg uncertainty relation in quantum mechanics sets the limit on the measurement precision of two conjugate observables. With squeezed states, one can measure one observable more precisely than the standard quantum limit at the expense of worse precision in the conjugate observable [1]. In some applications, however, we need to obtain the information embedded in two conjugate observables. For example, the real and imaginary parts of the linear susceptibility of an optical medium correspond respectively to the phase and amplitude modulation of an optical field passing through the medium. With the availability of entangled sources, it is possible to infer through Einstein-Podolsky-Rosen (EPR) correlation two conjugate observables with precision better than what is allowed by standard quantum limit [2-3]. In this paper, we implement a scheme to measure jointly the phase and amplitude modulation of an optical field in the newly developed SU(1,1) interferometer [4]. We achieve simultaneously a signal-to-noise ratio (SNR) improvement of 1.1 and $1 \mathrm{~dB}$ in amplitude and phase measurement over the standard quantum limit. Furthermore, because of the noise cancellation nature of the $\mathrm{SU}(1,1)$ interferometer [5], the signal-to-noise improvement occurs not only in simultaneous measurement of phase and amplitude but also in any two quadrature-phase amplitudes.

In contrast to the quantum dense coding scheme realized by using EPR entanglement (see Fig. 1(a)), out scheme shown in Fig. 1(b) employs an optical parametric amplifier to recombine the two entangled fields, replacing the 50/50 beam splitter (BS). This scheme is simply an SU $(1,1)$ interferometer [4]. For maximum sensitivity, the interferometer works at the dark fringe. As a result, destructive quantum interference leads to quantum noise cancelation, leading to minimum noise for all quadrature-phase amplitudes at the two outputs [4,5]. In the meantime, the input signals encoded by the phase modulator (PM) and amplitude modulator (AM) are amplified by OPA2 so the detected signal-to-noise ratios are increased for all quadrature-phase amplitudes as compared to the classical schemes without entangled state in Fig.1(c) and (d). According to our calculation, the measured SNRs of the amplitude and

This work is supported in part by National Natural Science Foundation of China (No. 11527808), the 973 project of China (No. 2014CB340103) and the National Key Research and Development Program of China (2016YFA0301403) phase modulations for the schemes in Figs. 1(b), 1(c) and 1(d) are expressed as:

$$
\begin{array}{ll}
S_{N N R_{S I}(\varepsilon s)=2\left(G_{1}+g_{1}\right)^{2} I_{p s} \varepsilon^{2}} & S_{N N R_{S I}(\delta i)=2\left(G_{1}+g_{1}\right)^{2} I_{p s} \delta^{2}} \\
S_{N N R_{B S}(\delta)=2 I_{p s} \delta^{2}} & S_{N R_{B S}(\varepsilon)=2 I_{p s} \varepsilon^{2}} \\
S N R_{A m}(\varepsilon s)=\frac{4 G^{2} I_{p s} \varepsilon^{2}}{\left(G^{2}+g^{2}\right)} & S N R_{A m}(\delta i)=\frac{4 g^{2} I_{p s} \delta^{2}}{\left(G^{2}+g^{2}\right)}
\end{array}
$$

where $\delta$ and $\varepsilon$ are the phase and amplitude modulations, respectively, and $\mathrm{I}_{\mathrm{ps}}$ is the photon number of the probe sensing field. The subscripts "SI" is for the SU(1,1) interferometer and "Am" is for the amplifier scheme in Fig. 1(c), which gives the same SNRs for joint measurement of $\delta$ and $\varepsilon$ for the BS scheme (see Fig. 1(d) when the gain of amplifier $G$ is very high. Notice that the SNRs for the SU(1,1) interferometer have a factor of $\left(\mathrm{G}_{1}+\mathrm{g}_{1}\right)^{2}$ improvement over the classical schemes in Figs. 1 (c) and 1(d).

(a)

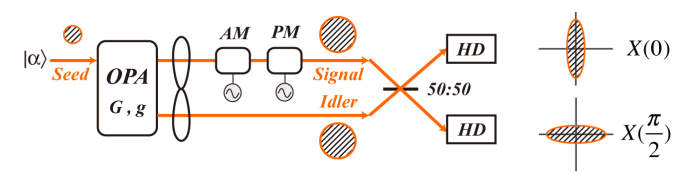

(b)

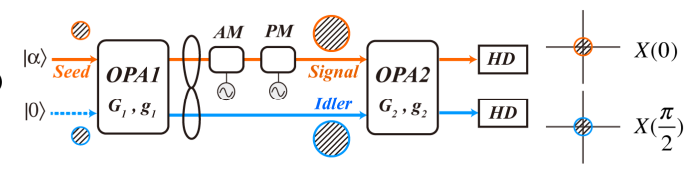

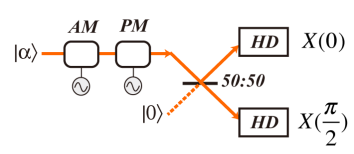

(c)

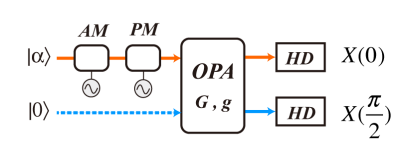

(d)
Fig. 1 Schematics for joint measurement of two conjugate observables. Modulation signals of amplitude and phase are encoded on the signal beam by an amplitude modulator (AM) and a phase modulator (PM). (a) Quantum dense coding scheme of utilizing EPR entangled sources to generating two squeezed states with noise reduced at two orthogonal quadrature-phase amplitudes. (b) An SU(1,1) interferometer with parametric amplifiers (OPA1, OPA2) as the equivalent beam splitters with noise reduction at all quadratures. (c) and (d) Classical schemes of joint measurement achieved by a beam splitter and by an amplifier. HD, homodyne detection.

In addition to the advantage over the classical scheme, the scheme with SU(1,1) interferometer has some more advantages over the scheme of quantum dense coding with a BS shown in Fig.1(a) [2-3]. Because the EPR entangled sources create via a 50:50 beam splitter two squeezed states with reduced noise at two orthogonal quadrature-phase amplitudes, the scheme in Fig.1(a) can only achieve joint measurement of two orthogonal 
observables such as the two conjugate quadrature-phase amplitudes: $\mathrm{X}$ and $\mathrm{Y}$ (equivalent to $\varepsilon$ and $\delta$ ). For the scheme with $\mathrm{SU}(1,1)$ interferometer, since the noise cancelation is done by the interferometer, the noise is reduced at all quadraturephase amplitudes [5]. So, for a modulation at an arbitrarily rotated quadrature-phase amplitude $X(\theta)=X \cos \theta+Y \sin \theta$, the SNR for $\mathrm{X}(\theta)$ is the same as $\mathrm{X}$ and $\mathrm{Y}$ when we measure it at either output port. Thus, the joint measurement can be performed on two arbitrary quadratures at the two outputs.

We conduct the joint measurement of $\varepsilon$ and $\delta$ with the scheme of SU(1,1) interferometer in Fig.1(b) and compare it with the classical scheme of beam splitting by a parametric amplifier (Fig.1(d)). This comparison is more direct than with the scheme in Fig.1(c) because both schemes in Fig.1(b) and (d) are involved with amplification of the modulated beam. The input information of amplitude and phase $(\varepsilon$ and $\delta$ ) are encoded on a beam in the input signal port of OPA2 by modulating the AM and PM with weak sinusoidal signal at 1.25 and $1.56 \mathrm{MHz}$, respectively. This beam is a classical coherent beam for the classical amplifier scheme in Fig.1(d) but is a quantum correlated beam from OPA1 for the scheme of SU(1,1) interferometer in Fig.1(b). The beam intensities are adjusted be to the same in order to have the same Ips for fair comparison. The amplifier gains are also same for the OPA in Fig.1(d) and the OPA2 in Fig.1(b) to ensure the same signal gain in the two cases. For the best sensitivity, the $\operatorname{SU}(1,1)$ interferometer is operated at dark fringe [4]. Simultaneous measurement of the two conjugate observables is achieved for both schemes by locking the relative phase of the homodyne detection (HD) measurements in signal and idler output ports at 0 and $\pi / 2$, respectively.

Figure 2 presents the joint measurement results. The blue traces in Figs. 2(a) and 2(b) are obtained with the $\operatorname{SU}(1,1)$ interferometer. The peaks of the blue traces in Figs. 2(a) and 2(b) are centering at 1.25 and $1.56 \mathrm{MHz}$, respectively, corresponding to an SNR of $5.4 \mathrm{~dB}$ and $4.8 \mathrm{~dB}$ for the two conjugate variables $\varepsilon$ and $\delta(\mathrm{X}(0)$ and $\mathrm{X}(\pi / 2))$, respectively. The red traces in Figs. 2(a) and 2(b) are obtained by setting the pump of OPA1 to zero so that OPA1 is not present. The SNRs of $\mathrm{X}(0)$ and $\mathrm{X}(\pi / 2)$ extracted from the red traces are $4.3 \mathrm{~dB}$ and $3.8 \mathrm{~dB}$, respectively. Comparing the results obtained by using the $\mathrm{SU}(1,1)$ interferometer and by using a conventional OPA, one sees that the peak heights of the red and blue traces are about the same. However, the noise floor of the blue traces is lower than that of the red traces by about 1.1 and $1 \mathrm{~dB}$ in Figs. 2(a) and 2(b), respectively, because the output noise of $\mathrm{SU}(1,1)$ interferometer is reduced by destructive quantum interference through quantum entanglement between the signal and the idler fields out of OPA1 [5]. So the improvement on SNRs in the joint measurement achieved by the $\mathrm{SU}(1,1)$ interferometer is obvious.
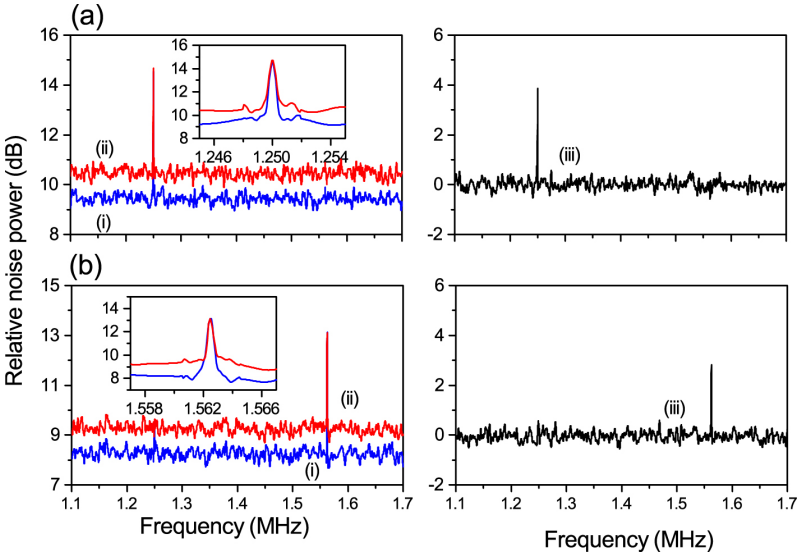

Fig. 2 Joint measurement of amplitude and phase $(x(0)$ and $x(\pi / 2)$ under different situations. (a) and (b) are signals simultaneously measured by locking the relative phase of HD to 0 and $\pi / 2$, respectively. Blues traces (labeled as (i)) are the results of scheme in Fig. 1(b). Red and black traces (labeled as (ii) and (iii)) are obtained by using classical methods in Figs. 1(d) and $1(\mathrm{c})$, respectively.

We also implement the beam splitting and direct measurement scheme of Fig.1(c). The results are shown as the black traces in Figs. 2(a) and 2(b), indicating the SNRs of measured $\mathrm{X}(0)$ and $\mathrm{X}(\pi / 2)$ are about $3.8 \mathrm{~dB}$ and $2.8 \mathrm{~dB}$ after the correction of the transmission efficiency. Ideally, the SNRs of joint measurement obtained by the two classical methods are the same. However, comparing the results of classical measurements, we find that the SNR of red trace is $0.5 \mathrm{~dB}$ better than that of the black trace. This is because the output noise of the conventional OPA is higher than the shot noise and is thus less sensitive to the vacuum noise due to detection loss than the direct homodyne measurement at shot noise level. This demonstrates another advantage besides the noise reduction for the joint measurement scheme of $\mathrm{SU}(1,1)$ interferometer which involves amplification before detection.

The experimental results for the joint measurement of two quadrature phase amplitudes, not necessarily orthogonal to each other will be presented at the conference.

\section{REFERENCES}

[1] M. Xiao, L. Wu and H. J. Kimble, "Precision measurement beyond the shot-noise limit," Phys. Rev. Lett. 59, 278 (1987).

[2] X. Li, Q. Pan, J. Jing, J. Zhang, C. Xie and K. Peng, "Quantum Dense Coding Exploiting a Bright Einstein-Podolsky-Rosen Beam," Phys. Rev. Lett. 8804790404 (2002).

[3] S. Steinlechner, J. Bauchrowitz, M. Meinders, H. M uller-Ebhardt, K. Danzmann, and R.Schnabel, "Quantum-dense metrology," Nat. Photon. 7, 626 (2013).

[4] F. Hudlist, J. Kong, C. Liu, J. Jing, Z. Y. Ou, and W. Zhang, "Quantum metrology with parametric amplifier-based photon correlation interferometers," Nature Comm. 5, 3049 (2014)..

[5] X. Guo, X. Li, N. Liu, Z. Y. Ou, "Quantum information tapping using a fiber optical parametric amplifier with noise figure improved by correlated input”, Sci. Rep., 6, 30214 (2016) 